[30] R. von ANgerer. Untersuchungen über die Ursachen der Resistenz von Bazillensporen. Arch. Hyg. Bakteriol., 121, 12 à 55.1939.

[31] O. B. Williams. The heat resistance of bacterial spores. J. inf. Dis., 44, 421 à 465 . 1929 .

\title{
RECHERCHES SUR LA FABRICATION, EN ROUMANIE, DU FROMAGE "TÉLÉMEA », DE LAIT DE BREBIS
}

\author{
par \\ Ing. Gheorghe Chintesco, Ing. Carol toma \\ et Ing. IOAN IONESCO \\ Institut de Recherches Alimentaires, Bucarest. R. P. R.
}

Parmi les fromages fabriqués dans notre pays, le fromage "Télémea sest le plus répandu et le plus apprécié par la masse des consommateurs.

La transformation de la petite production artisanale de ce fromage en une production industrielle a suscité une série de problèmes concernant la qualité de la matière première fournie aux centres de fabrication, ainsi que les différentes phases du processus technologique.

La quantité de lait de brebis produite dans notre pays augmente continuellement, d'où la nécessité d'outillages et d'installations appropriées. En ce cas, le processus technologique, obligé de s'adapter à la grande industrie, devra subir certaines modifications.

Tenant compte de.cette situation, nous nous sommes efforcés, au cours de nos recherches, de connaître les conditions de produetion du fromage "Télémea " fabriqué avec le lait de brebis dans la plaine et d'apprécier les facteurs ayant une influence défavorable sur la qualité du fromage, ainsi que sur les indices économiques.

Les caractéristiques physico-chimiques du lait de brebis servant à la fabrication du fromage "Télémea", ont également été déterminées.

Tenant compte des résultats obtenus par cette étude préliminaire, nous avons expérimenté un nouveau procédé de fabrication du fromage "Télémea ", qui présente un certain nombre d'avantages.

Afin d'améliorer les conditions de travail existantes, nous nous sommes servis de l'expérience acquise et des ourrages publiés en U.R.S.S. et en Bulgarie [1] [2]. 


\section{A. Conditions de la production du fromage «Télémea ». dans la plaine (Rég. de Constantza).}

Des travaux avaient été entrepris dans le but de déterminer certaines caractéristiques physico-chimiques de la matière première et d'étudier le processus technologique couramment employé pour la production dans l'une des fromageries de cette région, pendant la période 1 er juin-15 août.

1) Caractéristiques physico-chimiques du lait de brebis utilisé pour la fabrication du fromage "Télémea". Le lait de brebis provenait de trois bergeries situées à une distance de 7 à 10 kilomètres de la fromagerie. Il était transporté trois fois par jour, après chaque traite. Les résultats des analyses effectuées sont indiqués dans le tableau I, les chiffres représentant des données moyennes.

On a constaté que le lait de brebis utilisé présentait souvent beaucoup d'impuretés et une acidité élevée, ce qui a influencé défavorablement le processus de fabrication.

Cet état de choses est la conséquence des conditions de la traite et du ramassage, de la température ambiante élevée et de la trop longue durée du transport.

2) Le processus technologique expérimenté a été celui qu'on appliquait couramment dans les régions respectives. Le lait de chaque traite fut emprésuré séparément. On réalisait donc, journellement, trois fabrications. En tout quarante-deux fabrications.

On travaillait suivant le schéma technologique qui suit : réception qualitative et quantitative de la matière première, réglage de la température de coagulation $\left(27-30^{\circ} \mathrm{C}\right)$ - addition d'azotate de potassium (20-30 g pour 1001 de lait) - addition de présure coagulation (durée 40-60 minutes) - enlèvement du caillé et déposition sur une table d'égouttage - travail du caillé - pression (force de pression 0,4-o,8 kg/kg " cash " - salaison dans la saumure (10-16 heures, concentration $22 \%$ sel) — salaison sèche (6-12 heures) - emballage dans des récipients en bois - mise en dépôt dans des conditions naturelles (température $16-23^{\circ} \mathrm{C}$, durée maximum 45 jours).

En analysant la marche du processus de fabrication et les résultats obtenus, nous sommes arrivés aux constatations suivantes:

Vu la variabilité de la qualité de la matière première, le processus de fabrication ne se déroule pas toujours normalement; l'intense fermentation lactique accrô̂t considérablement l'acidité du fromage et le phénomène est parfois accompagné d'une fermentation spécifique des bactéries coliformes, ce qui fait diminuer la qualité du fromage et sa conservabilité. 
TABLEAU I

GARAGTÉristiques PHYSIGO-CHIMIQUes DU LAIT DE BREBIS UTILISÉ POUR LA FABRICATION DU FROMAGE (MOYENNES MENSUELLES)

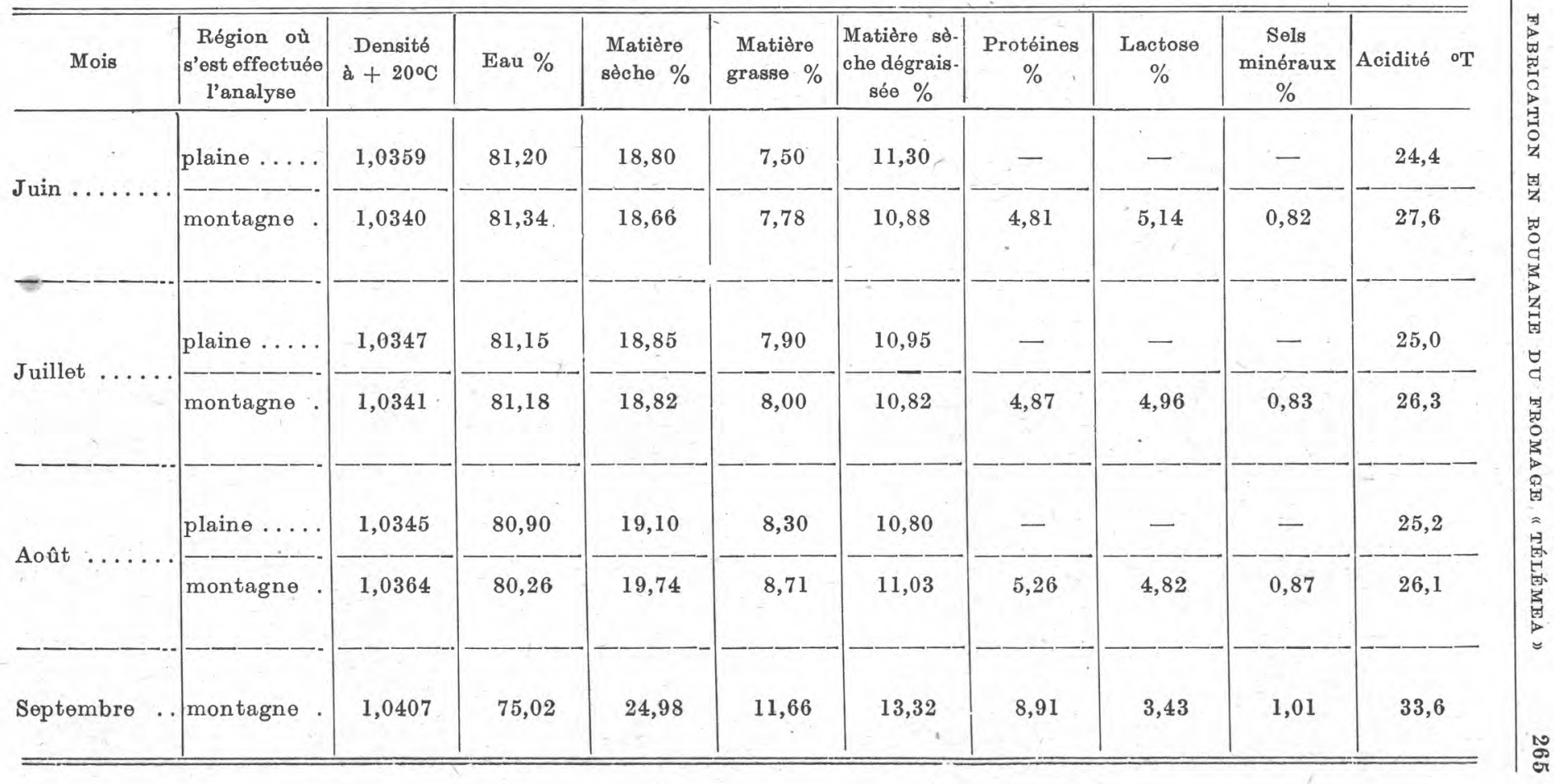


L'efficacité de l'azotate de potassium, en doses de 20-30 grammes pour 100 litres de lait, n'était pas constante, car le dessin qui se produit, lors de la présence des bactéries coliformes, apparaissait dans certaines fabrications.

Le rendement en fromage frais (tableau II) a été de $33,44 \%$.

TABLEAU II

RENDEMENT EN FROMAGE « TÉLÉMEA "

\begin{tabular}{|c|c|c|c|}
\hline \multirow{2}{*}{ Procédé de fabrication } & \multicolumn{2}{|c|}{$\begin{array}{l}\text { Quantité de fromage obtenue } \\
\text { à partir de } 100 \text { litres de lait }\end{array}$} & \multirow{2}{*}{$\begin{array}{c}\text { Pertes durant } \\
\text { la mise } \\
\text { en dépôt } \\
\%\end{array}$} \\
\hline & $\begin{array}{l}\text { Frais } \\
\mathrm{kg}\end{array}$ & $\begin{array}{l}\text { Maturé } \\
\mathrm{kg}\end{array}$ & \\
\hline Classique (régions de plaines). & 33,44 & 27,02 & 17,50 \\
\hline $\begin{array}{l}\text { Classique-témoin (région mon- } \\
\text { tagneuse) } \ldots \ldots \ldots \ldots \ldots \ldots\end{array}$ & 34,48 & 30,95 & 9,91 \\
\hline $\begin{array}{c}\text { Expérimental (région monta- } \\
\text { gneuse) } \ldots \ldots \ldots \ldots \ldots \ldots\end{array}$ & 32,78 & 29,15 & 10,92 \\
\hline
\end{tabular}

Vingt à trente jours après la mise en dépôt, à une température de 16 à $23^{\circ} \mathrm{C}$., le rendement varia entre 23,57 et 31,84 (en moyenne 27,02). Les valeurs les plus élevées furent obtenues au cours du mois d'août, le lait ayant acquis à cette époque un pourcentage élevé en matière sèche. Le rendement plus faible du fromage mûri (après 20-30 jours) peut être attribué, entre autres, aux pertes en poids subies pendant la période de mise en dépôt. Vingt à trente jours après la mise en dépôt, ces pertes atteignirent les valeurs les plus élevées - 17,50\% ; après cet intervalle et jusqu'au quarantecinquième jour, les pertes en poids furent assez peu importantes. La composition chimique moyenne du fromage mûri est indiquée dans le tableau III.

Afin d'améliorer la qualité du fromage "Télémea », on jugea nécessaire - à part l'organisation et la dotation technique des fromageries - d'introduire la pasteurisation de lait de brebis et d'employer certaines cultures spécifiques de bactéries lactiques, ce qui a rendu possible de diriger le processus de maturation du fromage. Certaines modifications de la technologie classique s'imposent d'ailleurs en vue de réduire la durée de la fabrication et de créer des possibilités de mécaniser la production. 
TABLEAU III

GOMPOSITION CHIMIQUE DU FROMAGE "TÉLEMEA " MATURĚ (CHIFFRES : MOYENNES)

\begin{tabular}{|c|c|c|c|}
\hline \multirow[t]{3}{*}{$\cdot-$} & $\begin{array}{c}\text { Région } \\
\text { de plaines }\end{array}$ & \multicolumn{2}{|c|}{ Région montagneuse } \\
\hline & \multicolumn{3}{|c|}{ Procédé de fabrication } \\
\hline & Classique & $\begin{array}{l}\text { Classique- } \\
\text { témoin }\end{array}$ & $\begin{array}{l}\text { Expérimental } \\
\text { «en formes }\end{array}$ \\
\hline Matière sèche \% ....... & 52,15 & 49,77 & 48,93 \\
\hline Matière grasse $\%$........ & 27,12 & 24,86 & 24,16 \\
\hline Matière grasse rapportée à & & . & \\
\hline la substance sèche $\%$.. & 52,00 & 49,74 & 49,49 \\
\hline Protéines $\% \ldots \ldots \ldots \ldots$ & - & 19,23 & 19,01 \\
\hline Chlorure de sodium $\% \ldots$ & 6,5 & 5,5 & 6,4 \\
\hline Acidité en ${ }^{\circ} \mathbf{T} \ldots \ldots \ldots \ldots$ & 159 & 129 & 108 \\
\hline Pointage $\ldots \ldots \ldots \ldots \ldots$ & 83 & 92 & 87 \\
\hline
\end{tabular}

Dans les régions du pays à climat chaud, comme par exemple la région de Constantza, le fromage doit être transporté immédiatement après sa préparation dans des frigorifiques, où les conditions de température et d'humidité nécessaires à un processus normal de maturation, auront été créées. Quant au rendement, il serait recommandable de le déterminer 20 à 30 jours après la préparation, alors que les pertes en poids deviennent à peu près constantes.

\section{B. Expérimentation d'un nouveau procédé de fabrication du fromage «Télémea ».}

En tenant compte des résultats obtenus dans la première partie de ce travail, on étudia, dans une région montagneuse du pays (Azuga) :

a) les caractéristiques physico-chimiques du lait de brebis utilisé pour la fabrication du fromage ;

b) la fabrication du fromage "Télémea " du lait de brebis pasteurisé, auquel on ajoute un levain de yoghourt selon le procédé classique de fabrication ;

c) la fabrication du fromage "Télémea" suivant le nouveau procédé dit « en formes».

Caractéristiques physico-chimiques de la matière première: le lait de brebis utilisé pour la fabrication, fut procuré par quatre bergeries coopératives. L'intervalle entre la traite et l'arrivée du lait à 
la fromagerie, varia entre 2 et 6 heures. On procéda à l'analyse du lait de brebis provenant de la traite du matin, de celle de midi et de celle du soir, pendant la période $1^{\text {er }}$ juin-17 septembre.

Les valeurs moyennes mensuelles des éléments composant la matière première ont été indiquées dans le tableau I. En analysant ces valeurs, on constate que les modifications les plus évidentes apparaissent au cours du mois de septembre, quand tous les éléments constitutifs du lait de brebis, excepté la lactose, atteignent, par rapport au mois de juin, leur valeur la plus haute.

Si l'on compare la variation de la teneur en protéines à celle de la teneur en matière grasse, il résulte que cette dernière présente une augmentation plus importante durant les premiers mois, tandis que pendant le dernier mois, cette augmentation est dépassée par celle des protéines. Pour toute la période étudiée, la variation de la teneur en substances protéiques (augmentation $=4,10 \%$ ) est plus grande par rapport à celle du contenu en matières grasses (augmentation $=3,82 \%$ ).

Il faut remarquer que - à mesure que la période de lactation est plus avancée - le pourcentage de lactose du lait de brebis baisse, tandis que la teneur en autres éléments constitutifs du lait s'élève. - Par suite de la variabilité de l'acidité du lait de brebis pendant la période de lactation et surtout pendant le mois de septembre, la détermination de l'acidité titrable ne donne pas toujours des indications concernant le degré de fraîcheur du lait.

\section{Expérimentation du procédé de fabrication "en formes"}

On exécuta séparément, le même jour, des fabrications expérimentales avec du lait provenant de chaque traite. Le lait utilisé pour la préparation, fut préalablement pasteurisé à $65^{\circ} \mathrm{C}$. pendant 30 minutes, puis refroidi à la température de coagulation. Les quantités de lait pasteurisé furent partagées en deux, le premier lot constituant la matière première pour la fabrication du fromage " Télémea" au moyen du procédé classique, le second étant soumis au nouveau procédé expérimental dit “en formes ».

Le principe de cette méthode [3] réside dans le découpage et la division du caillé dans la chaudière jusqu'à l'obtention d'une certaine dimension du " grain ", en réalisant par cela une certainedéshydratation partielle de celui-ci. La masse du caillé ainsi préparée est mise dans des formes métalliques, composées de deux corps, ayant chacun 6 compartiments (fig. 1), disposés sur des plaques métal'liques pourvues de sillons pour l'égouttage du sérum (petit-lait).

Un certain temps après la mise en forme du caillé, celui-ci, par auto-compression, pénètre complètement dans la forme intérieure. 
On enlève alors la partie supérieure de la forme ; puis, afin d'assurer l'égouttage du petit-lait, on retourne les formes trois ou quatre fois pendant 40 à 60 minutes.

Fig. 1.

Forme métallique pour le fromage "Télémea ».

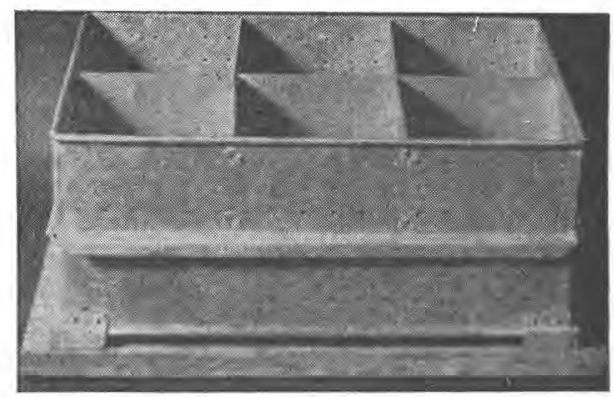

Le tableau IV montre les phases principales du processus de fabrication, suivant l'un et l'autre des procédés.

Les expériences effectuées ont conduit aux conclusions suivantes :

L'action du levain de yoghourt se manifeste de manière différente. Dans le cas des fabrications du type "en formes", on enregistre une acidité plus réduite du fromage, par rapport aux échantillons-témoins, phénomène dû surtout à la durée plus courte de préparation.

A cause du travail du caillé dans la chaudière et de sa mise en formes, les fromages préparés par le nouveau procédé subissent une pulvérisation prononcée du caillé et de plus grandes pertes de matière grasse dans le petit-lait, par comparaison aux fromages de fabrication classique.

La teneur en matière grasse du petit-lait provenant des fabrications expérimentales " en formes " est en moyenne de 1,25\%, par rapport à celle $(0,59 \%)$ enregistrée pour les fabrications-témoin.

En comparant la durée de la préparation des deux procédés, on constate que le procédé "en formes» s'avère plus avantageux, sa durée proprement dite (jusqu'à la salaison) étant réduite de deux heures.

Les deux lots de fromage se différencient au point de vue organoleptique. Par le fait de travailler le caillé " en graine », le fromage " en formes " acquiert une structure différente. En section, il présente de rares espaces vides (diamètre $3-10 \mathrm{~mm}$.), dus à la disposituion des particules de caillé, qui déterminèrent une répartition non homogène de l'eau dans la masse du fromage. Sa consistance fut plus molle, son goût et son arome, quoique normaux, sont moins expressifs par rapport aux échantillons-témoins. 
TABLEAU IV

PRINGIPALES PHASES DES DEUX PROCÉdÉS DE FABRIGATION DU FROMAGE * TÉLEMEA "

Processus de fabrication

\begin{tabular}{l}
\hline Classique \\
\hline $\begin{array}{l}\text { Pasteurisation du lait à } 65^{\circ} \mathrm{C} . \\
(30 \text { minutes }) .\end{array}$
\end{tabular}

Refroidissement du lait à la température de coagulation 28-31 ${ }^{\circ} \mathrm{C}$.

Addition de levain 0,5-0,8\%.

Addition de $\mathrm{CaCl}_{2}$ en proportion de 15-20 grammes pour 100 litres de lait, et présure.

Coagulation du lait durant 4560 minutes.

Enlèvement du caillé de la chaudière à l'aide d'une écumoire et mise sur un tamis placé sur la table d'égouttage.

Premier découpage du caillé en morceaux prismatiques ayant 4 centimètres de côté.

Repos pendant 10-20 minutes.

Deuxième découpage du caillé en prismes ayant 3 centimètres de côté.

Repos pendant $30-40$ minutes.

Coupe des bords et empaquetage du "Cash " en forme d'enveloppe.

Pression. Durée 2 heures, force de pression initiale 65 kilogrammes, finale 130 kilogrammes pour $100 \mathrm{ki}$ logrammes de fromage.
Expérimental "en formes *

Pasteurisation du lait à $65^{\circ} \mathrm{C}$. (30 minutes).

Refroidissement du lait à la tem pérature de coagulation de $32-33^{\circ} \mathrm{C}$.

Addition de levain 0,5-0,8\%.

Addition de $\mathrm{CaCl}_{2}$ en proportion de 15-20 grammes pour 100 litres de lait, et présure.

Coagulation du lait durant 20-30 minutes.

Découpage et division du caillé dans la chaudière.

Division des particules de caillé jusqu'à obtention d'un grain de $1 \mathrm{~cm} .5$ de diamètre (20-25 minutes).

Repos pendant 5 minutes.

Extraction de $25 \%$ du sérum de la chaudière.

Le caillé préparé est placé dans des formes métalliques.

Auto-pression : les formes sont retournées 4 fois à des intervalles de 10-15 minutes.

Salaison dans la saumure (concentration $22 \%$ de sel) durant 16 20 heures. 
TABLEAU IV (suite)

\begin{tabular}{l}
\hline Classique \\
Coupe du Cash en morceaux \\
prismatiques, côté de la base = \\
15 centimètres.
\end{tabular}

Les morceaux sont placés sur la table d'égouttage pendant 10-15 minutes.

Salaison dans la saumure (concentr. sel. 22-24\%) durant $16-18$ heures.

Salaison sèche durant 4-8 heures.

Emballage dans des récipients en bois.

Mise en dépôt.
Expérimental "en formes »

Salaison sèche durant 4-6 heures.

Emballage dans des récipients en bois.

Mise en dépôt.

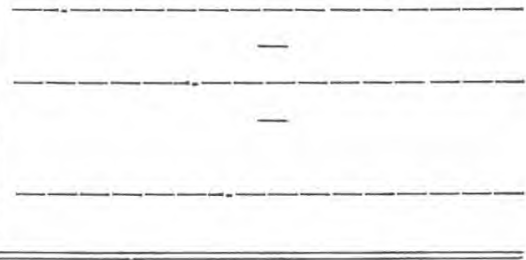

Ces derniers présentent des caractéristiques normales; la teneur élevée en sel, pour les deux échantillons, a une influence défavorable sur le goût et l'arome du fromage.

Les fromages fabriqués à l'aide du nouveau procédé présentent, après trois à quatre mois de mise en dépôt, une consistance plus dure à l'intérieur qu'à l'extérieur. Le goût devient plus piquant, indiquant l'accélération des processus de fermentation.

La composition chimique moyenne des fromages fabriqués par l'un et l'autre des procédés est analogue (tableau III). Les méthodes employées pour l'analyse ont été celles indiquées dans la littérature [4].

Le rendement en fromage cru, dans les deux cas, ne présente pas de différences notables (tableau II). Après une période de maturation de 30 jours, le rendement diminue chez les lots expérimentaux, par rapport aux échantillons-témoins $(29,15$ contre 30.95$)$.

A la suite des travaux exécutés dans la phase expérimentale, certaines conclusions se détachent, concernant les avantages et les désavantages du nouveau procédé de fabrication, par rapport au procédé classique.

\section{Avantages}

- La durée proprement dite du processus de fabrication (jusqu'à la salaison) est de 3 heures au lieu de 5 (procédé classique). 
Elle se trouve done réduite de $40 \%$ ).

- Certaines phases du processus de fabrication, difficilement applicables dans la production industrielle du fromage "Télémea ", sont simplifiées ou même éliminées.

- En réduisant la durée de fabrication, on évite l'acidification du lait, surtout pendant la saison chaude; cela permet l'emploi d'une quantité de lait accrue en un temps plus court.

- L'application du nouveau procédé offre des possibilités de mécaniser certaines phases du processus de fabrication : le découpage et la division du caillé, l'enlèvement du caillé, la mise en formes, le renversement des formes, etc.

- Les morceaux de fromage sont homogènes, ayant une composition analogue à celle du fromage préparé selon la méthode clas. sique.

\section{Désavantages}

- Des déchets pulvérulents de caillé se produisent en plus grande mesure et la quantité de matière grasse passant dans le petitlait est plus grande que pour le fromage fabriqué par le procédé classique. Ces facteurs contribuent à diminuer le rendement.

- Les fromages "en formes" ont une structure et des caractéristiques organoleptiques différentes par rapport aux fromages préparés selon la méthode classique. En même temps, leur conservabilité semble moindre que celle des fromages de fabrication classique.

Les importants avantages que le nouveau procédé de fabrication présente, malgré tout, pour la production industrielle du fromage "Télémea ", nous ont déterminé à continuer les recherches.

Les travaux seront dirigés en vue d'apporter certaines modifications au processus expérimental de fabrication, modifications qui doivent contribuer à l'amélioration des qualités organoleptiques, à l'augmentation du rendement et de la conservabilité du fromage obtenu par ce nouveau procédé.

\section{SUMMARY}

The importance of "Télémea " cheese produced from ewe's milk has occasioned research techniques resulting in a better applied technology : pasteurization, addition of lactic cultures, preparation after separation of the curdled milk.

The advantages and disadvantages brought about by the applied modifications were noted upon completion of the experiments and are explained here.

The advantages obtained justify the continuation of the research studies. 


\title{
BIBLIOGRAPHIE
}

[1] M. K. Scvortzov. Proizvodstvo brinzi. 1951.

[2] N. Drmov, I. Salicev. La valorisation du lait de brebis (trad. du bulgare. Edit. de l'Etat pour la littérature agricole, Bucarest, 1957). P. 116 (62).

[3] H. Dermisek. Fabrication du fromage Télémea "en formes» (1956). La Revue de l'Industrie Alimentaire pour les Produits Animaux, $\mathrm{n}^{\circ} 1, \mathrm{p} .21$.

[4] G. Inihov, N. Brio. Analyse chimique des produits laitiers (trad. du russe, I. D. T., 1953). P. 530 (62).

\section{CONTRIBUTION A L'ÉTUDE DES MÉTHODES DE RECHERCHE DES NEUTRALISANTS DANS LES POUDRES DE LAIT ÉCRÉMÉ}

\author{
(Fin) \\ par
}

L. SERRES, S. AMARIGLIO, L. BENARD, D. ROBINET

Laboratoire Central du Service Technique Interprofessionnel du Lait

\section{II. - ÉTUde GOMPARÉ DES RÉSUltats DES MÉthodes APPLIQUEES AUX LAITS LIQUIDES \\ ET AUX POUDRES CORRESPONDANTES}

Le but de nos travaux étant la recherche et le dosage des neutralisants dans les poudres de lait, nous avons comparé les résultats des méthodes d'analyse précédentes appliquées au lait d'une part, à la poudre obtenue à partir de ce même lait dans les conditions du séchage industriel d'autre part.

La dessication du lait par chauffage plus ou moins fort risque de modifier certains composants du lait, surtout dans les poudres fabriquées sèlon le procédé Hatmaker; en effet, le lait est soumis à une augmentation brusque de température, il prend une coloration légèrement crème et, parfois, certaines particules sont brûlées.

Par ailleurs, les variations intéressant les teneurs des principaux constituants (matière azotée, lactose, qui forment la plus grande part de l'extrait sec du lait écrémé, donc de la poudre) ne sont pas nécessairement parallèles à celles des constituants organiques (acide lactique,...) ou minéraux $(\mathrm{K}, \mathrm{Na}, \ldots)$ dont la détermination sert de base à la recherche des neutralisants.

Govld [58] a montré que le chauffage du lait écrémé augmente la quantité d'acides organiques (formique et acétique); d'après Grimbleby [59], les acides formés de 100 à $116^{\circ} \mathrm{C}$. (lactique et formique) sont compensés par une perte en acides volatils. 